\title{
The Non-linear Chromatic Compensation of the Fixed Length Fiber's Spectral Attenuation with the Fourier Transform
}

\author{
Xianjiang Meng \\ College of Communication Science and Engineering \\ Shenyang Ligong University \\ Shenyang,China \\ mengxj@jlu.edu.cn
}

\begin{abstract}
In the chromatic measuring system, a kind of optical fiber probe was added to collect the light to decrease the interference to the reflected light from the object. But the probe brings the distortion to the system in the chromatic measurement. This paper put forward a method based on the Fourier Transform to compensate the non-linear attenuation of the fixed length optical fiber. In the scope of visible light, with a series of experiments, an adjustable function for the optical fiber probe is obtained. Using this adjustable function, the error of fiber-optic transmission can be lowed less than $1 \%$. At the same time, the chromatic coordinate was tested with the fiber probe and without the probe. The error of the chromatic coordinate is below 0.01 , and it meets the international standard criteria in chromatic measurement. It is proved that this method is suitable for compensating of the fixed length optical fiber's chromatic compensation.
\end{abstract}

Keywords-optical fiber probe;spectral attenuation; the Fourier Transform; Chromatic Compensation; chromatic coordinate

\section{INTRODUCTION}

The fiber-optic probe has the characteristics of high anti-interference, good concentration in energy transmission, high sensitivity and low price. It can be used in measuring system without touching the object. It has a great variety of applications in sensors [1-3]. Its non-linear characteristics also has been discussed that it has different methods to compensate when the fiber-optic probe is used as a sensor in spectrum analysis [4-8]. Now we will use it in chromatic measurement field. But the probe has different absorption to the power light according the wavelength. The spectrum out from the probe is very different from the input, and this will bring distortion to the chromatic value. So it is very important to compensate the power loss of the fiber-optic probe according the wavelength[9].

\section{THE TESTING SYSTEM}

The testing system is shown in Fig .1, the light source irradiates straightly into the grating monochromator when we get the spectrum without the fiber-optic probe, but when we get the spectrum with the fiber-optic probe, we let the light go through the probe, reflected by the standard white plate, and then into grating monochromator. The fiber-optic probe, which is taken as light-collecting system, brings the distortion to the system. The grating in the monochromator is driven by the step motor, the light is

\author{
Xianli Meng \\ Heilongjiang Branch \\ China Mobile Group Telecommunication Design \\ Institute Co., Ltd \\ Harbin, China \\ kissnoy@sina.com
}

diffracted by the grating. The diffracted light is received by the window of the photomultiplier, and then the signal is converted into the current. The current was amplified and converted into digital signal by $\mathrm{A} / \mathrm{D}$, and then put into computer for data processing. In the paper, the data were obtained in the wavelength from $380 \mathrm{~nm}$ to $780 \mathrm{~nm}$. The standard A light source is a bromide-tungsten light, the maximum light power is $50 \mathrm{~W}$, the data was sampled every $5 \mathrm{~nm}$. It confirms to the request of the international chromatic measurement.

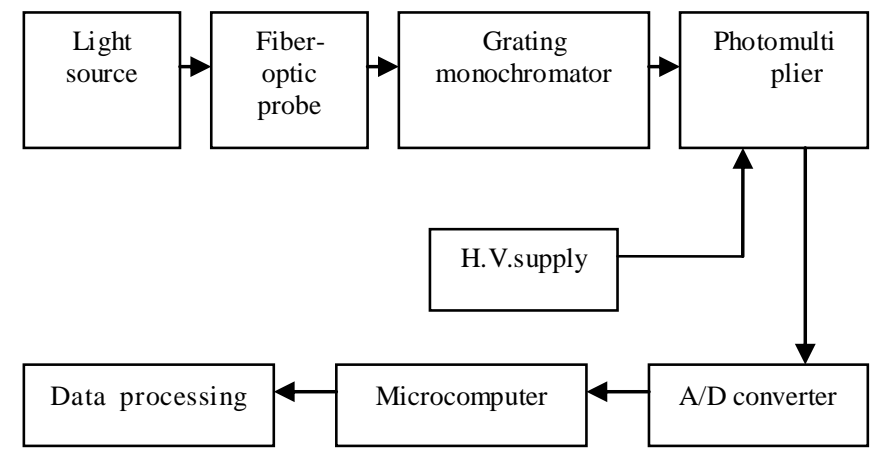

Figure 1. The framework chart of the testing system

A bromide-tungsten light (color temperature is $2856 \mathrm{~K}$ ) was used as standard light source; the grating monochromator and photomultiplier were used as the spectrum analyzing system [10]. The measurement was accomplished at the wavelength $380-780 \mathrm{~nm}$, It is proved by the experiment that the attenuation of the fiber-optic probe was compensated very well.

\section{THE PRINCIPLE OF COMPENSATION}

A. The structure of the optical fiber probe

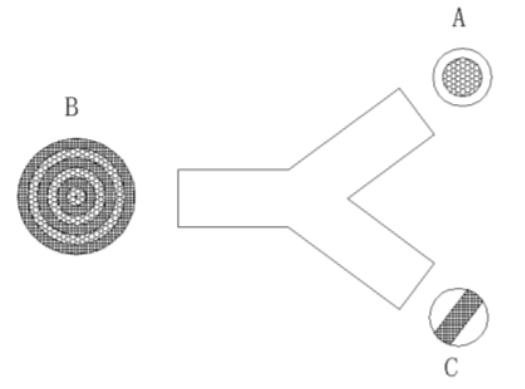

Figure 2. The structure of the optical fiber probe 
The structure of the optical fiber probe is shown in Fig .2. The fiber-optic probe consists of three parts: input port $\mathrm{A}$, output part $\mathrm{C}$ and coupled part $\mathrm{B}$. The probe is made of a large variety of stepped multi-mode fiber. The fiber is FG105UCA, wavelength range is $250-1200 \mathrm{~nm}$. The diameter of the core is $105 \pm 2 \mathrm{um}, \mathrm{NA}=0.22 \pm 0.02$. The diameter of port $\mathrm{A}$ and $\mathrm{B}$ is $0.5 \mathrm{~cm}$ and $0.8 \mathrm{~cm}$ respectively, the port $\mathrm{C}$ is a $0.3 \mathrm{~cm} * 1.2 \mathrm{~cm}$ rectangular, the leg is $70 \mathrm{~cm}$ in length. The input port $\mathrm{A}$ is a cylindrical shape attached to the light source. The port A is just located at the focus, the light irritates from the light source is reflected by a concave mirror directly into port $\mathrm{A}$ and then into port B. The pot B is made of the input fiber and output fiber interchangeably. The input light is reflected almost perpendicularly into output fiber and out from port $\mathrm{C}$. The port $\mathrm{C}$ is made into a slit shape in order to match the input port of the monochromator, the port $\mathrm{C}$ is fastened to the slit through a mechanical device to ensure the tight fit, and make sure the most light energy coupled into the monochromator.

\section{B. The testing system}

The testing system is devised as shown in Fig .3. The spectrum of the light source with the fiber-optic probe and without it is shown in Fig .4. In the chart we can see the crest of the spectrum did not move, only the spectrum away from the crest attenuated, and the more away from the crest, the more attenuation it produced. So the spectrum output from the probe is sharper. In Fig .4, the spectrum output from the fiber-optic probe has a bigger value in the higher frequency of the Fourier spectrum than the one without fiber-optic probe. That is to say the higher frequency increases through the probe. So we can compensate the distortion by weakening the spectrum at the higher frequency.

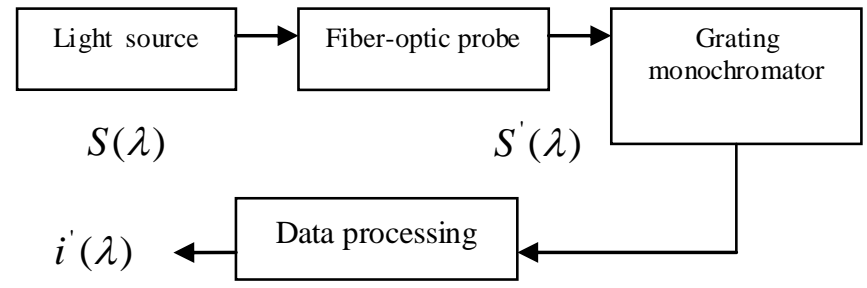

Figure 3. The scheme of the testing system

IV. The ADJUSTING PRINCIPLE

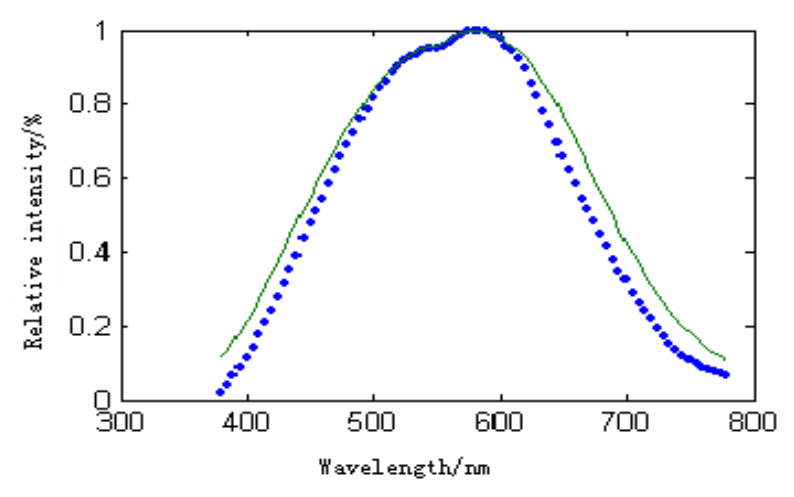

... with fiber-optic probe; —without fiber-optic probe

Figure 4. The light source spectrum with and without the fiberoptic probe

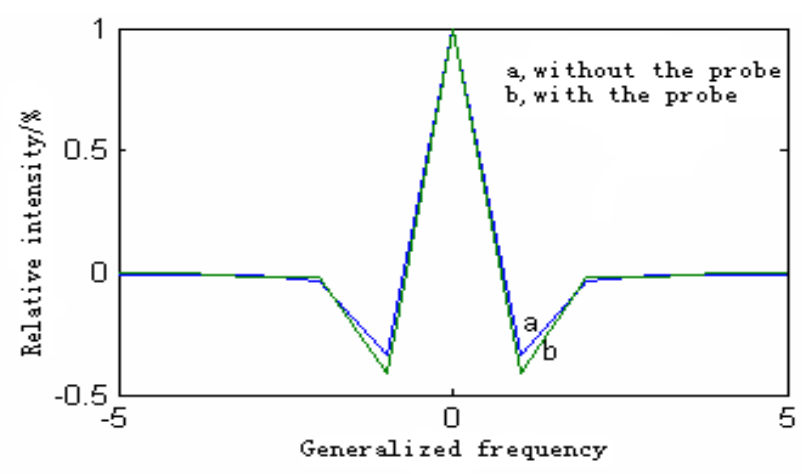

Figure 5. The difference of the Fourier frequency chart with and without the fiber-optic probe

To achieve the compensation on the attenuation of fiber-optic probe in generalized spectrum of frequency, suppose the spectrum of the light source is $S(\lambda)$, when measuring without the fiber-optic probe, the output current is

$$
i(\lambda)=k(\lambda) \times S(\lambda)
$$

$k(\lambda)$, a factor, it reflects the influence of the grating monochromator to the system.

Suppose the output spectrum of light source spectrum from the probe is $S^{\prime}(\lambda)$, when measuring with the fiber-optic probe, the output current is

$$
i^{\prime}(\lambda)=k(\lambda) \times S^{\prime}(\lambda)
$$

The two kinds of the output currents are transformed with Fourier Transform, their spectrum lines of frequency are

$$
\begin{aligned}
& F(f)=\int_{-\infty}^{+\infty} i(\lambda) \exp (-i 2 \pi f \lambda) d \lambda \\
& F^{\prime}(f)=\int_{-\infty}^{+\infty} i^{\prime}(\lambda) \exp (-i 2 \pi f \lambda) d \lambda
\end{aligned}
$$

Then the adjusting function to the spectrum with the probe in frequency domain is

$$
\Delta F(f)=T(f) *\left[F^{\prime}(f)-F(f)\right]
$$

The Formula (5) was transformed with Fourier Transform, so the adjusting function in the light domain is

$$
\Delta i(\lambda)=\int_{-\infty}^{+\infty} \Delta F(f) \exp (i 2 \pi f \lambda) d \lambda
$$

$T(f)$, is the Fourier Transform of the penetrating ratio of the measured object, to the light source, $T(f) \equiv 1$; to the ideal optical filters, it is the Fourier Transform of the penetrating ratio function, calculate the inverse Fourier Transform on both side of the formula, we get the adjusting function $T(f)$.

Suppose $j^{\prime}(\lambda)$ is the spectrum reflected from the measured object with the probe, the adjusting is

$$
j(\lambda)=j^{\prime}(\lambda)-\Delta i(\lambda)
$$

$$
j(\lambda) \text {, is the spectrum after adjusting. }
$$


TABLE I. THE CHARACTERISTIC WAVELENGTH/NM

\begin{tabular}{|c|c|c|c|c|c|c|}
\hline $\begin{array}{c}\text { characteristic } \\
\text { wavelength }\end{array}$ & $\begin{array}{c}\text { measured } \\
\text { value 1 }\end{array}$ & $\begin{array}{c}\text { measured } \\
\text { value 2 }\end{array}$ & $\begin{array}{c}\text { measured } \\
\text { value 3 }\end{array}$ & $\begin{array}{c}\text { measured } \\
\text { value 4 }\end{array}$ & $\begin{array}{c}\text { measured } \\
\text { value5 }\end{array}$ & $\begin{array}{c}\text { Average } \\
\text { value }\end{array}$ \\
\hline 404.7 & 404.8 & 404.7 & 404.6 & 404.9 & 404.7 & 0.07 \\
\hline 435.8 & 435.8 & 435.7 & 435.6 & 435.7 & 435.7 & 0.10 \\
\hline 546.1 & 546.3 & 546.1 & 546.2 & 546.2 & 546.1 & 0.10 \\
\hline 577.0 & 577.0 & 577.1 & 577.0 & 576.9 & 577.1 & 0.03 \\
\hline 579.0 & 579.1 & 579.2 & 579.0 & 579.1 & 579.1 & 0.10 \\
\hline
\end{tabular}

\section{DATA PROCESSING AND ANALYSIS}

\section{A. The measuring system wavelength calibration}

The accuracy of the wavelength setting is referred to the deviation of the showed wavelength with the actual wavelength by the mechanical process error or the motor inertia and so on. All sorts of factors cause the inaccuracy of the measured spectrum, and influence the accuracy of the system severely. In the GB/T3979-2008, to the wavelength instrument, the tolerance of the standard wavelength is no more than $0.5 \mathrm{~nm}$. In calibration it is formal to use the mercury spectrum lamp as light source, and control the stepped motor to scan manually. In experiment, we measure the characteristic wavelengths of mercury lamp to analyze: $404.7 \mathrm{~nm}, 435.8 \mathrm{~nm}, 546.1 \mathrm{~nm}, 577.0 \mathrm{~nm}$ and $579.0 \mathrm{~nm}$, in order to check the wavelength deviation of the system. The measured values are shown in Table I.

It is can be seen from Table I, the average error of characteristic wavelengths of mercury lamp is $0.1 \mathrm{~nm} \sim 0.166 \mathrm{~nm}$, the single testing error is less than $0.2 \mathrm{~nm}$, it confirms to request of the international standard wavelength error $0.5 \mathrm{~nm}$.

\section{B. The average adjusting function and spectral compensation}

To get a function to compensate the attenuation of the fiber-optic probe, we can get the average of multimeasurement. In this paper, we got five groups of spectrum data with fiber-optic probe and without it respectively. For decreasing the error of data measurement with the probe, we got the adjusting function for the light source with the average of the five groups data, the adjusting function is shown in Fig .6. Similarly we got the adjusting functions of three kinds of the light filters: red, yellow and green.

\section{The Fourier Transform adjusting}

We can use the Fourier Transform to adjust the compensation of the fiber-optic probe precisely. In this method, the current is changed into the spectrum domain, in the spectrum domain we get the deviation, and then is inverse transformed to the current domain; an adjusting function is obtained as in Formula (7). we used the adjust function to compensate the distorted spectrum of light source with the fiber-optic probe. The result is shown in
Fig .7. We can see the spectrum after adjusting with probe and without probe is very similar. The maximum

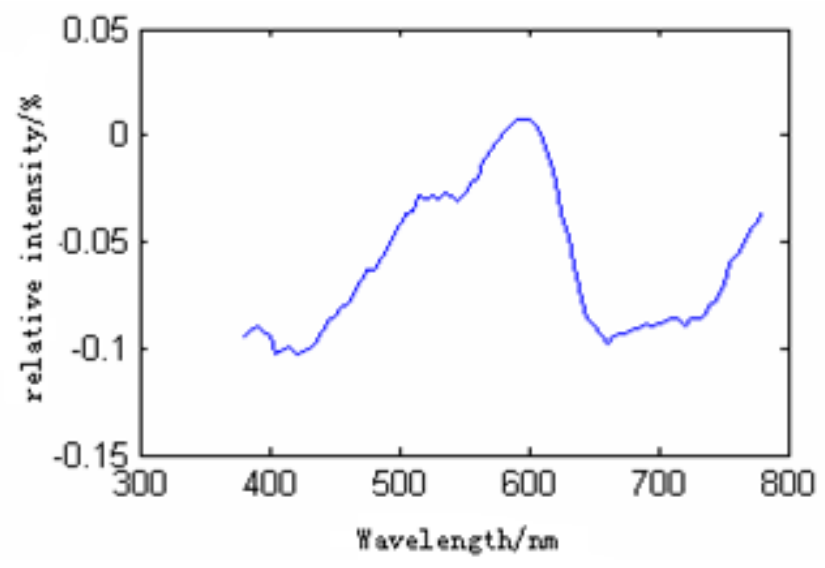

Figure 6. The average adjusting function of the light source

absolute error is 0.0093 ; the average absolute error is 0.0058 . So the maximum absolute error is below $1 \%$.

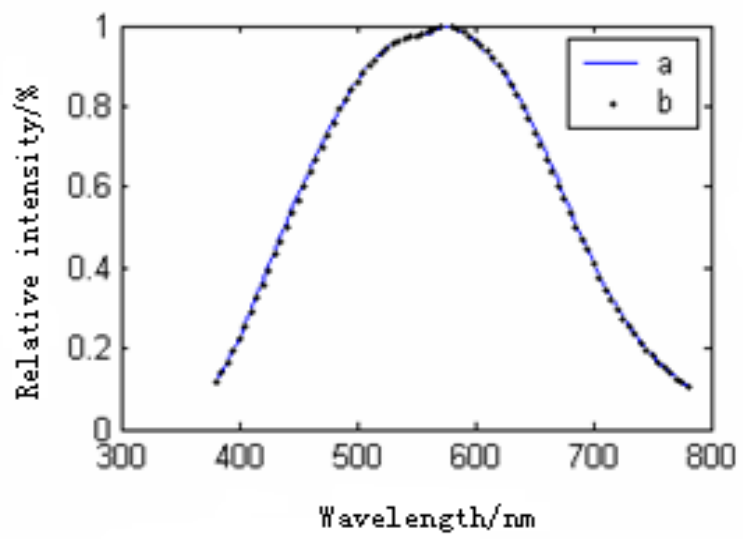

Figure 7. The light source spectrum after compensation

In Fig .8, we got the three adjusting functions of the three kinds of light filters, and the spectrum after adjusting is shown in Fig .9. The maximum absolute error is 0.0092 (red), 0.0095 (yellow) and 0.0098(yellow); the average absolute error is 0.0053 (red), 0.0055 (yellow) and 0.0056 (green). So the maximum error is also below $1 \%$, we get a good compensation performance. 


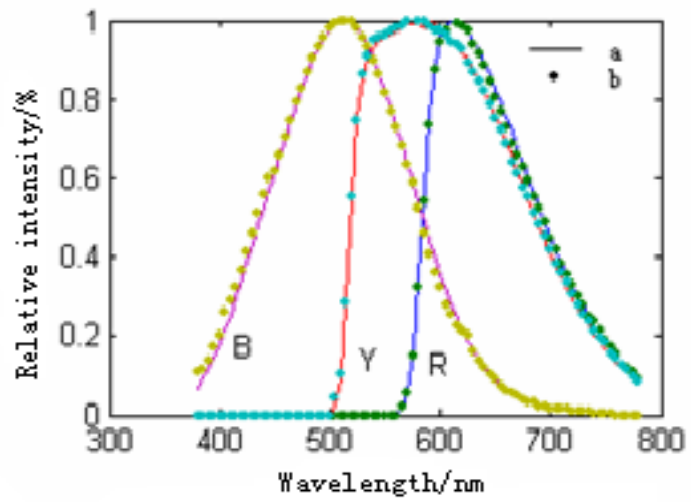

a, spectrum without the fiber-optic probe;

$\mathrm{b}$, adjusted spectrum with the fiber-optic probe;

$\mathrm{R}$, red filter; $\mathrm{Y}$, yellow filter; $\mathrm{B}$, blue filter

Figure 8 . The spectrum of the three filters after compensation

\section{The measuring system chromatic precision}

Chromaticity coordinate is the most important parameter to the comprehensive evaluation of the chromatic system. This paper measure the precision of the use chromaticity coordinate by measuring three standard color reflected cards. The chromaticity coordinate of the standard color reflected cards is measured by LAMBDA9UV/VIS/NIR spectrometer from PETRKIN-ELMER

TABLE II. THE CHROMATICITY COORDINATE VALUE company, America, which belongs the state key laboratory of applied optics of Changchun Institute of Optics and Fine Mechanics, The chromaticity coordinate precision is 0.001 .

First the light from the light source irradiate the different color reflected cards, the reflected light goes directly into monochromator. The spectrum is measured and then chromaticity calculated. Many measured value and average measured are listed in TableII.

Then the light from the light source irradiate the different color reflected cards, the reflected light goes through the fiber-optic probe and then into monochromator, he spectrum is measured and then Chromaticity calculated according formula (3). Many measured value and average measured are listed in Table II.

From Table II, it can be seen the fiber-optic probe is added into the system, the chromaticity coordinate error is larger than 0.01 and it cannot satisfy the request of chromaticity measurement. Through the chromatic compensation with the Fourier transform, the average error of the chromaticity coordinate error is 0.005 and the largest error 0.0090 , so the chromaticity coordinate error is below 0.01 and it meets international standards of the error of the chromaticity coordinate error is less than 0.01 . From the analysis above, the chromatic precision is improved greatly.

\begin{tabular}{|c|c|c|c|c|c|c|c|c|c|c|c|c|}
\hline \multirow{3}{*}{ No. } & \multicolumn{6}{|c|}{ the chromaticity coordinate value without probe } & \multicolumn{6}{|c|}{ the chromaticity coordinate value with probe } \\
\hline & \multicolumn{2}{|c|}{ Red card } & \multicolumn{2}{|c|}{ Yellow card } & \multicolumn{2}{|c|}{ Green card } & \multicolumn{2}{|c|}{ Red card } & \multicolumn{2}{|c|}{ Yellow card } & \multicolumn{2}{|c|}{ Green card } \\
\hline & $\mathrm{x}$ & $\mathrm{y}$ & $\mathrm{x}$ & $\mathrm{y}$ & $\mathrm{x}$ & $\mathrm{y}$ & $\mathrm{x}$ & $\mathrm{y}$ & $\mathrm{x}$ & $\mathrm{y}$ & $\mathrm{x}$ & $\mathrm{y}$ \\
\hline 1 & 0.6242 & 0.3473 & & 0.4496 & 0.3869 & 0.5315 & 0.6189 & 0.3485 & 0.5312 & 0.4491 & 0.3988 & 0.5156 \\
\hline 2 & 0.6238 & 0.3471 & & 0.4430 & 0.3889 & 0.5294 & 0.6203 & 0.3498 & 0.5296 & 0.4471 & 0.3976 & 0.5175 \\
\hline 3 & 0.6234 & 0.3471 & & 0.4467 & 0.3892 & 0.5299 & 0.6211 & 0.3520 & 0.5323 & 0.4436 & 0.4030 & 0.5125 \\
\hline 4 & 0.6238 & 0.3482 & & 0.4498 & 0.3881 & 0.5295 & 0.6194 & 0.3521 & 0.5299 & 0.4507 & 0.3998 & 0.5123 \\
\hline 5 & 0.6256 & 0.3469 & & 0.4520 & 0.3878 & 0.5314 & 0.6207 & 0.3556 & 0.5312 & 0.4521 & 0.4001 & 0.5144 \\
\hline 6 & 0.6249 & 0.3488 & & 0.4511 & 0.3872 & 0.5301 & 0.6159 & 0.3538 & 0.5321 & 0.4485 & 0.4059 & 0.5140 \\
\hline Standard & 0.6142 & 0.3575 & 0.5350 & 0.4483 & 0.4022 & 0.5116 & 0.6142 & 0.3575 & 0.5350 & 0.4483 & 0.4022 & 0.5116 \\
\hline $\begin{array}{c}\text { Average } \\
\text { error }\end{array}$ & 0.0102 & 0.0099 & 0.0091 & 0.0027 & 0.0091 & 0.0187 & 0.0052 & 0.0055 & 0.0038 & 0.0002 & 0.0008 & 0.0028 \\
\hline $\begin{array}{c}\text { Maximu } \\
\text { m error }\end{array}$ & 0.0123 & 0.0106 & 0.0112 & 0.0053 & 0.0112 & 0.0199 & 0.0069 & 0.0090 & 0.0054 & 0.0038 & 0.0046 & 0.0059 \\
\hline
\end{tabular}

\section{CONCLUSION}

In the traditional method, we usually use the ratio of the spectrum with and without the probe as the adjusting function. In this paper, we put forward a kind of method to adjustment of the attenuation of the probe with Fourier transform. The maximum absolute error is 0.0093; the maximum chromatic coordinate error is 0.009 , it is below the international standard 0.01 . The average absolute error is 0.0058 ; the maximum absolute error is below $1 \%$. From the adjusting effect above, we found the method with Fourier transform can get a better effect in compensating the attenuation. It is much better than the traditional method in compensation of the fiber-optic probe attenuation. So this method has a good performance and can be put into applications of spectral analysis and chromatic measurement.

\section{ACKNOWLEDGMENT}

This paper is funded by the National Nature and Science Fund of China. Thanks to my workmate PHD

Weijie Shi's cooperation.

\section{REFERENCES}

[1] Wang Yongxin,Han Ming, Wang Anbo,High-speed fiber-optic spectrometer for signal demodulation of inteferometric fiber-optic sensors,Optics Letters, Vol. 31, pp.2408-2410 „July 2006

[2] M.Taki;F. Zaidi;I.Toccafondo,High-performance hybrid Raman/fiber Bragg grating fiber-optic sensor based on simplex cyclic pulse coding,Optics Letters, Vol. 38, pp.471-473,April 2013

[3] Abad, Silvia,Araújo, Transparent Network for Hybrid 
Multiplexing of Fiber Bragg Gratings and Intensity-Modulated FiberOptic Sensors, Applied Optics, Vol. 42, pp.5040-5045,August 2003

[4] G. Bobo,Y. Wu, Frosz, Nonlinear fiber-optic strain sensor based on four-wave mixing in microstructured optical fiber,Optics Letters, Vol. 37 pp.794-796,May 2012

[5] Kim, Kwang-Taek; Park,Fiber-Optic Temperature Sensor Based on Single Mode ,Journal of the Optical Society of Korea, Vol. 12, pp.152-156,March 2008

[6] Shokin, I.Yu,O.Shtyrina, Mathematical modelling of nonlinear effects in optical fiber,Russian Journal of Numerical Analysis and Mathematical Modelling, Vol.25,P93-104,January 2010

[7] B.Tiwari,V.Prakash, V. Tripathi Nonlinear Effects in Optical Fiber Transmission System,IETE TECHNICAL REVIEW, Vol.16,P461480,May 1999
[8] Shiva Kumar,Dong Yang,Optical backpropagation for fiber-optic communications using highly nonlinear fibers,Optics Letters,01469592, VOL.36,P1038-1040.July 2011

[9] T.Inoue,O. Aso,S. Namiki,Recent Issues on Nonlinear Effects in Optical Fibers,ACTA OPTICA SINICA, Vol.23,P37-39, October 2007

[10] P. West,M.Bostrom,P.Torzilli, Fourier Transform Infrared Spectral Analysis of Degenerative Cartilage: An Infrared Fiber Optic Probe and Imaging Study,Applied Spectroscopy, Vol.58, P376-381,April 2009

[11] Tariq A.K. Al-Jumaily,Dispersion Compensation in Optical Fibers Communication System Using Spectral Inversion,Journal of Engineering and Applied Sciences, 1816-949,Vol.6,p387-389,June 2011

[12] William W. Parson, Modern Optical Spectroscopy, Springer ,March 2008 